\title{
VISUALIZATION OF FRINGES IN TIME AVERAGED MOIRE PATTERNS
}

\author{
Loreta Saunorienė, Minvydas Ragulskis \\ Department of Mathematical Research in Systems, Kaunas University of Technology \\ Studentu St. 50, LT-51368 Kaunas, Lithuania
}

\begin{abstract}
Time averaging interferometry is an experimental technique widely applied in different areas of optical engineering. Typical example is an application of time averaging techniques for the analysis of fringes produced by a moiré grating formed on the surface of oscillating elastic structure. Unfortunately, time averaged moiré produces blurred images. Therefore, special digital image filtering is to be used for highlighting the pattern of time averaged fringes. Such a procedure is essential for the investigation of dynamical systems with non-harmonic motion as the produced moiré pattern is particularly blurred. This paper proposes some techniques for the sharpening of fringes in time averaged moiré patterns.
\end{abstract}

Keywords: moiré, digital image, visualization, morphological operators.

\section{Introduction}

Double exposure geometric moiré $[5,9]$ is an experimental technique based on analysis of visual patterns produced by superposition of two periodic gratings that geometrically interfere. Moiré techniques are widely applied in different areas of optical engineering. Deformations are measured directly from the moiré image by interpreting the produced fringes $[1,6]$. The contrast of the obtained fringe pattern in double exposure moiré image is sufficient enough and no extra visualization techniques are required. Moiré grating is formed on the surface of elastic oscillating structure for analysis of dynamic displacements and time averaging techniques are used for the formation of fringe patterns [10,11]. Nevertheless, the produced time averaged moiré image is not as sharp as image produced by double exposure geometric moiré. Therefore, special digital image filtering must be used for highlighting the pattern of time averaged fringes. This procedure is essential for the investigation of dynamical systems with non-harmonic motion as the produced moiré pattern is then particularly blurred. The paper introduces some techniques which can be effective for the sharpening of the fringes in moiré patterns. Additionally, morphological operations are adapted for the removal of initial moiré grating irrelevant for the interpretation of fringes.

\section{Geometric moiré}

One-dimensional example is analysed for simplicity. Moiré grating formed on the surface of one dimensional structure in the state of equilibrium is interpreted as harmonic function $[5,10]$

$$
I_{1}(x)=\cos ^{2}\left(\frac{\pi}{\lambda} x\right),
$$

where $\lambda-$ is the pitch of grating, $x$ - geometrical coordinate. Values of $I_{1}$ vary in the range between 0 (black colour) and 1 (white colour) (Figure 1(a)).

Moiré grating in the deformed state can be expressed in the following way (Figure 1(b)) [10]:

$$
I_{2}(x)=\cos ^{2}\left(\frac{\pi}{\lambda}(x-u(x))\right),
$$

where $u(x)$ stands for displacement from the state of equilibrium at point $x$.

The intensity of illumination of double exposure geometric moiré $I_{d}$ is calculated as the arithmetic mean of intensity functions $I_{1}$ and $I_{2}$ (Figure 1(c)) [11]:

$$
\begin{aligned}
& I_{d}(x)=\frac{1}{2}\left(\cos ^{2}\left(\frac{\pi}{\lambda} x\right)+\cos ^{2}\left(\frac{\pi}{\lambda}(x-u(x))\right)\right)= \\
& =\frac{1}{2}+\frac{1}{2} \cos \left(\frac{2 \pi}{\lambda}\left(1-\frac{u(x)}{2 x}\right) x\right) \cos \left(\frac{\pi}{\lambda} u(x)\right) .
\end{aligned}
$$

The centres of fringes are located at such point $x$ where the branches of the envelope function $\frac{1}{2} \pm \frac{1}{2} \cos \left(\frac{\pi}{\lambda} u(x)\right)$ intersect [11]: 


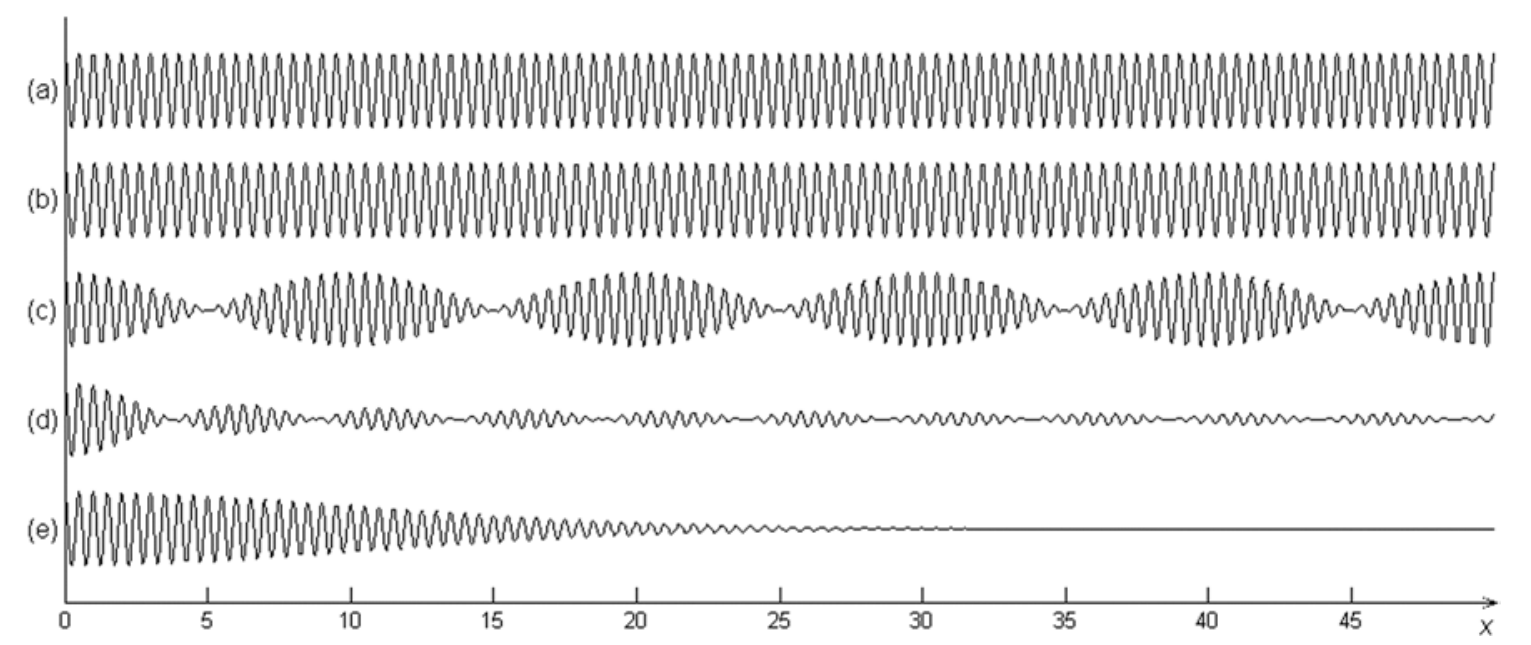

Figure 1. Intensity of illumination: (a) $I_{1}(x)$; (b) $I_{2}(x)$; (c) $I_{d}(x)$; (d) $I_{t}^{h}(x)$; (e) $I_{h}^{s}(x)$, when $u(x)=k x ; \lambda=0.5 ; k=0.05 ; \sigma=0.15$

$$
u(x)=\lambda\left(n-\frac{1}{2}\right)
$$

Figure 1(c) shows that the produced fringes are sufficiently clear and there is no demand to increase the sharpness of the image.

\section{Time averaged geometric moiré}

Double exposure geometric moiré techniques provide insight into static in-plane displacements of analysed bodies. Time averaged geometric moiré is a technique applicable for the analysis of oscillating elastic structures [10].

If the deformation of the grating from the state of equilibrium (eq.(2)) oscillates in time, the intensity of illumination at point $x$ and at time moment $t$ can be described as:

$$
I_{1}(x-u(x) \cdot \zeta(t)),
$$

where $\zeta(t)$ is a function determining the variation of displacement $u(x)$ in time.

If time averaging techniques are used for the determination of intensity of the surface (and the exposure time is much longer than the frequency of oscillations), then the averaged intensity of illumination at point $x$ is:

$$
I_{t}(x)=\lim _{T \rightarrow \infty} \frac{1}{T} \int_{0}^{T} \cos ^{2}\left(\frac{\pi}{\lambda}(x-u(x) \cdot \zeta(t))\right) d t,
$$

where $T$-exposure time.

Let's assume that the analysed structure oscillates harmonically with angular frequency $\omega$ and phase $\varphi$ : $\zeta(t)=\sin (\omega t+\varphi)$. Then the intensity of time averaged moiré $I_{t}^{h}$ at any point $x$ can be expressed [11]:

$$
I_{t}^{h}(x)=\frac{1}{2}+\frac{1}{2} \cos \left(\frac{2 \pi}{\lambda} x\right) J_{0}\left(\frac{2 \pi}{\lambda} u(x)\right),
$$

where $u(x)$ defines the amplitude of dynamic displacement and $J_{0}$ is zero order Bessel function of the first kind:

$$
J_{0}(a)=\lim _{T \rightarrow \infty} \frac{1}{T} \int_{0}^{T} \exp (\mathrm{j} a \sin (\omega t-\varphi)) d t .
$$

Fringes are modulated by the envelope function $E_{t}^{h}(x)=\frac{1}{2} \pm J_{0}\left(\frac{2 \pi}{\lambda} u(x)\right) \quad$ (Figure 1(d)). The displacement of the point $x$ located at the centre of the $n$-th fringe can be calculated [11]:

$$
u(x)=\frac{\lambda}{2 \pi} r_{n},
$$

where $r_{n}$ is the $n$-th root of the zero order Bessel function of the first kind.

It can be noted that time averaged fringes do not carry any information about frequency or phase of oscillations.

It can be observed that time averaged moiré image is blurred if compared with double exposure geometric moiré (Figure 1(d)). Digital image filtering techniques must be applied for fringe visualization. It is especially relevant for the fringes of higher orders.

Equation (7) determines the intensity of time averaged fringe pattern if only oscillations of the sample are harmonic. How will the fringes be modulated if $\zeta(t)$ is treated as a non-harmonic process? Let's assume that $\zeta(t)$ is approximated by a discrete series of random normally distributed numbers with zero mean and $\sigma^{2}$ variance: $\zeta_{i} \sim N\left(0, \sigma^{2}\right)$. It can be shown [8] that intensity of time averaged moiré image is then: 


$$
I_{t}^{S}(x)=\frac{1}{2}+\frac{1}{2} \cos \left(\frac{2 \pi}{\lambda} x\right) \cdot e^{-\frac{1}{2}\left(\frac{2 \pi}{\lambda} u(x) \sigma\right)^{2}} .
$$

Fringe pattern is modulated by the envelope function:

$$
E_{t}^{s}(x)=\frac{1}{2} \pm \frac{1}{2} \cdot e^{-\frac{1}{2}\left(\frac{2 \pi}{\lambda} u(x) \sigma\right)^{2}} .
$$

It can be noted that no fringes will be produced at all (Figure 1(e)) and intensity converges exponentially to 0.5 for large displacements. The rate of convergence is conditioned by the value of variance. The greater value of variance results in the faster convergence.

\section{Filtering techniques}

Let's assume that the intensity of the original twodimensional image is $I(x, y)$. Then $F(I(x, y))$ denotes the intensity of the highlighted image.

How should one choose the function $F$ to sharpen the pattern of interference fringes? Intensity of illumination of original image $I(x, y)$ varies between 0 (black colour) and 1 (white colour); besides, the centres of fringes correspond to intensity of $I(x, y)=0.5$. These statements lead to the following properties of filtering function $F$ :

- $F$ is symmetrical in respect to the intensity level $I(x, y)=0.5: F(I(x, y))=F(1-I(x, y))$,

- $F \in[0 ; \delta]$ if and only if $I(x, y) \in[0.5-\varepsilon ; 0.5+\varepsilon]$,

where $\varepsilon$ and $\delta$ are small positive numbers. The digital image is darkened where the intensity is around 0.5 $(0.5-\varepsilon<I(x, y)<0.5+\varepsilon)$ and is brightened elsewhere.

The following functions for the filtering of the digital image are proposed.

1. $F(I(x, y))=\tanh ^{2}(k(I(x, y)-0.5))$.

The curves of this function are presented in Figure 2 for various values of $k(k=1,2, \ldots, 10)$. Parameter $k$ defines the depth of darkening of the digital image around $I=0.5$. Small values of $k$ produce too wide fringes and insufficient contrast between fringes and the whole image. At $k<1$ the whole image is darkened. Very large $k$ generates thin unsmooth fringes; moreover, the obtained image is bleached.

2. $F(I(x, y))=\left\{\begin{array}{l}\frac{1}{k} \cdot|I(x, y)-0.5| \\ \text { if } 0.5-k \leq I(x, y) \leq 0.5+k, \\ 1, \text { otherwise. }\end{array}\right.$

The shape of this function is demonstrated in Figure 3 (at $k=0.2$ ). The sharpness of the fringes for large parameter $k$ is poor; the fringe itself is rather wide. Thin unsmooth fringes and bleached image is obtained if parameter $k$ is relatively small.
3. $F(I(x, y))= \begin{cases}0, & 0.5-k \leq I(x, y) \leq 0.5+k, \\ 1, & \text { otherwise. }\end{cases}$

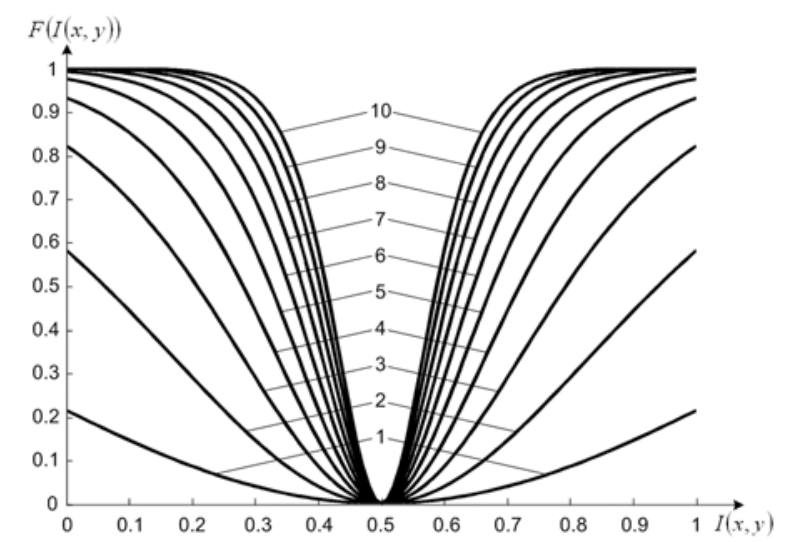

Figure 2. $F(I(x, y))=\tanh ^{2}(k(I(x, y)-0.5))$; where $k=1,2, \ldots, 10$

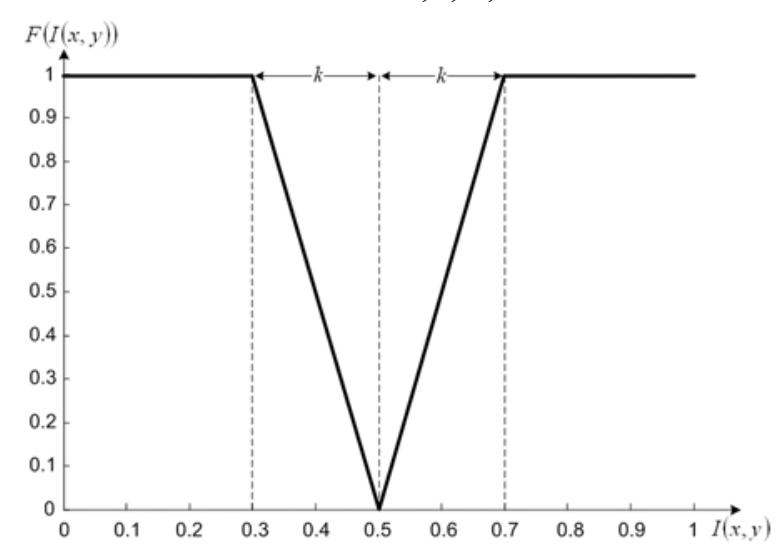

Figure 3. $F(I(x, y))=\left\{\begin{array}{l}\frac{1}{k} \cdot|I(x, y)-0.5| \\ \text { if } 0.5-k \leq I(x, y) \leq 0.5+k, \\ 1, \text { otherwise. }\end{array}\right.$

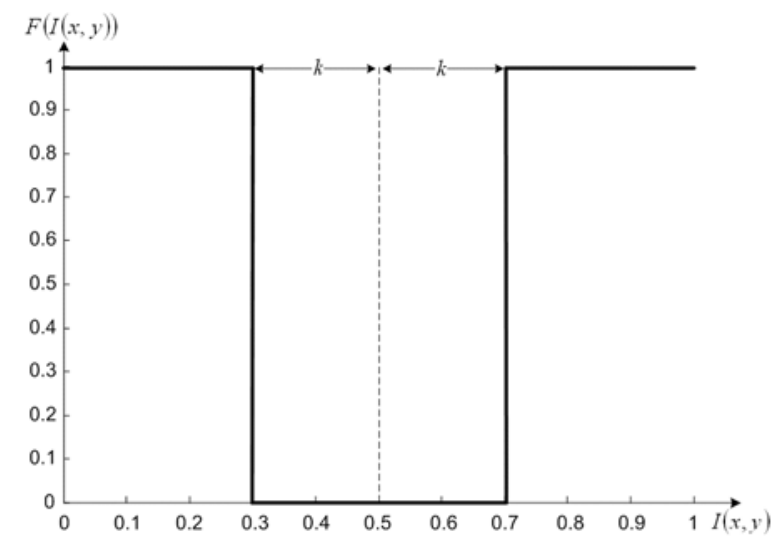

Figure 4. $F(I(x, y))= \begin{cases}0, & 0.5-k \leq I(x, y) \leq 0.5+k \\ 1, & \text { otherwise }\end{cases}$

An illustration of this threshold function is given in Figure 4. Function (14) produces a binary image while the result of functions (12) and (13) is a greyscale representation. 
Filtering functions help to highlight fringe pattern but they make no effect on original moiré grating which is irrelevant for the interpretation of fringes. This grating can be removed by applying morphological operators to the digital image $[2,3,4]$. Morphological operators are

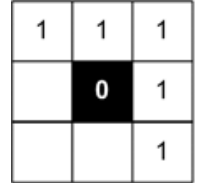

\begin{tabular}{|l|l|l|}
\hline 1 & 1 & 1 \\
\hline 1 & 0 & \\
\hline 1 & & \\
\hline
\end{tabular}

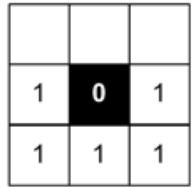

(a)

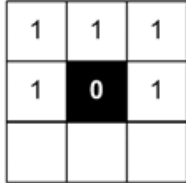

particularly used for the analysis of binary images though their application to greyscale images is also possible.

Figure 5. Structuring elements: (a) for hit and miss transformation; (b) for erosion operation

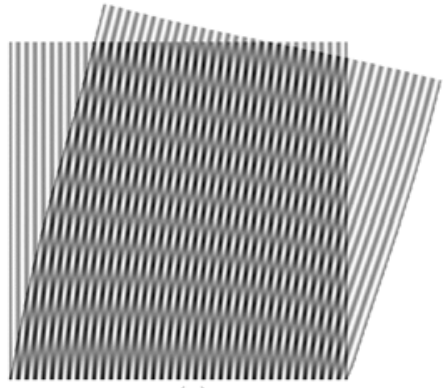

(a)

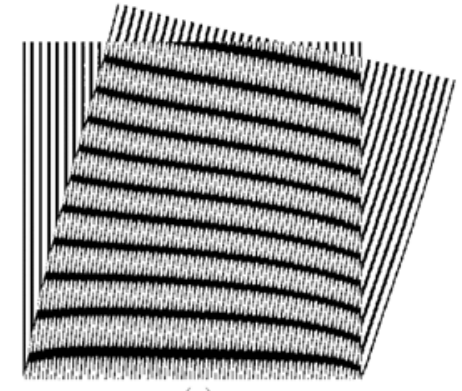

(b)

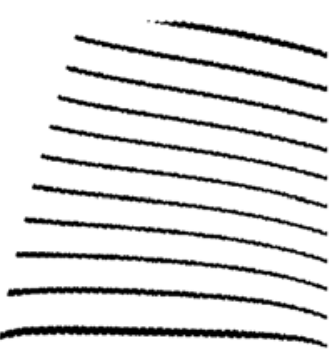

(c)

Figure 6. Visualization of double exposure geometrical moiré: (a) original image; (b) highlighted image; (c) highlighted and cleaned image

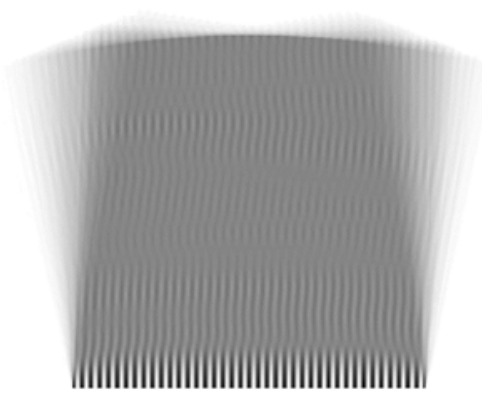

(a)

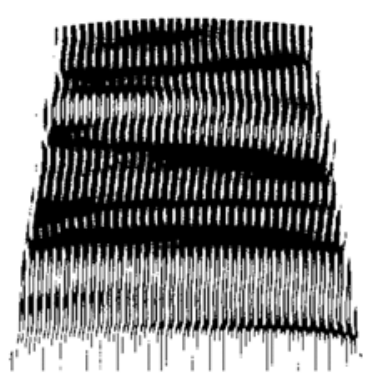

(b)

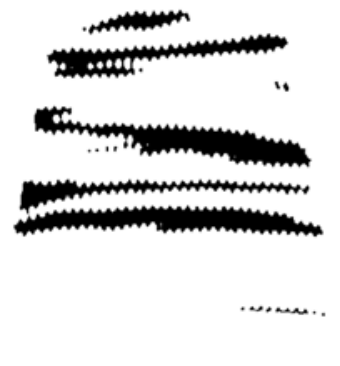

(c)

Figure 7. Visualization of time averaged geometrical moiré: (a) original image;

(b) highlighted image; (c) highlighted and cleaned image

Morphological operators use a binary image and a structuring element as input and combine them using a set operator (intersection, union, etc.). Usually, the structuring element is a matrix sized $3 \times 3$ and has its origin at the centre pixel $[2,4]$. It is shifted over the image and its elements are compared with the set of the underlying pixels. If the two sets of elements match the condition defined by the set operator, the pixel underneath the origin of the structuring element is set to a pre-defined value ( 0 or 1 for binary images) [4].

The lines of original moiré grating have several black pixels in width so foreground pixels with a particular number of background neighbours must be deleted for their removal. Hit and miss transformation [4] is appropriate in this case as it can be used to look for several patterns (or, alternatively, for the same pattern in several orientations).

Several versions of structuring element for hit and miss transformation are presented in Figure 5(a). Surely, other variations of structuring element are also possible. Foreground pixel (black) is set to background colour (white) when its neighbours match pixels in the matrix of structuring element exactly. The number of background pixels $n_{f}$ in the structuring element depends upon the width of original grating lines. The thinner are the grating lines, the greater is the parameter $n_{f}$.

Erosion operator [4] is an alternative for hit and miss transformation. In the following example, $3 \times 3$ structuring element (Figure 5(b)) is used. The goal of 
this operation is to remove any foreground pixel that is not completely surrounded by other black pixels.

The grating is removed by iterating hit and miss or erosion operations for several times. High iteration numbers can degrade the fringes. Furthermore, the whole image can be completely deleted in both cases.

\section{Example for visualization of fringes}

Visualization of fringes in double exposure geometric moiré image is presented in Figure 6. The threshold function (eq.(14)) at $k=0.15$ and the erosion operator (Figure 5(b)) are used for the image processing.

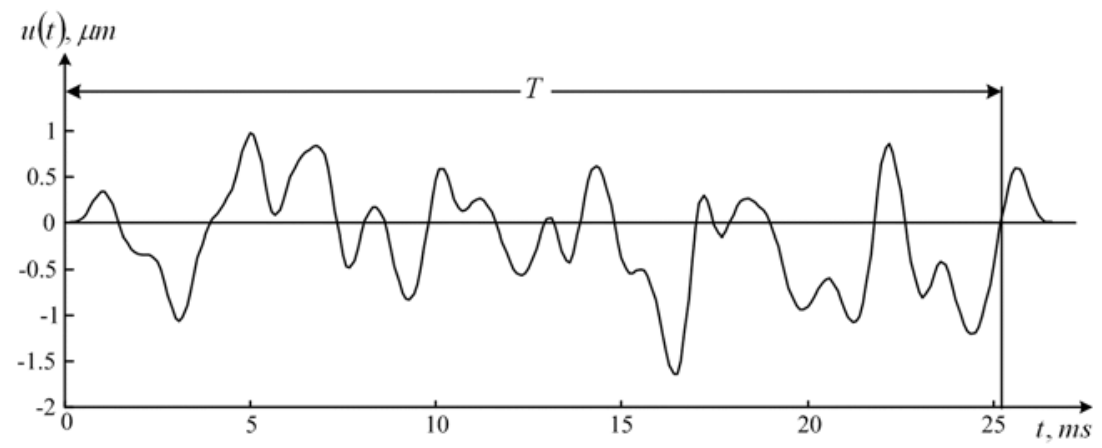

Figure 8. Chaotic oscillations of the dynamical system over exposure time $T$

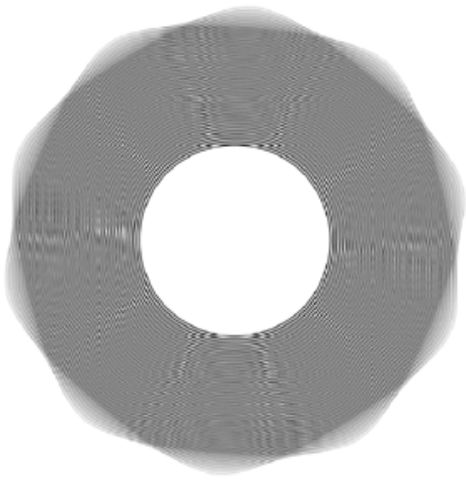

(a)

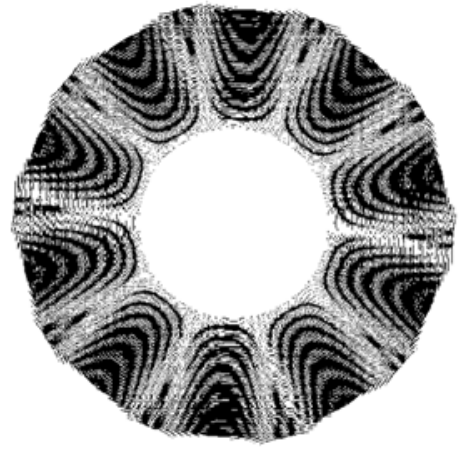

(b)

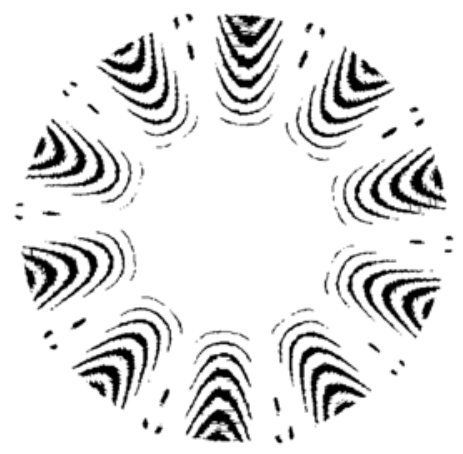

(c)

Figure 9. Visualization of time averaged reflection moiré (harmonic oscillations): (a) original image; (b) highlighted image; (c) highlighted and cleaned image

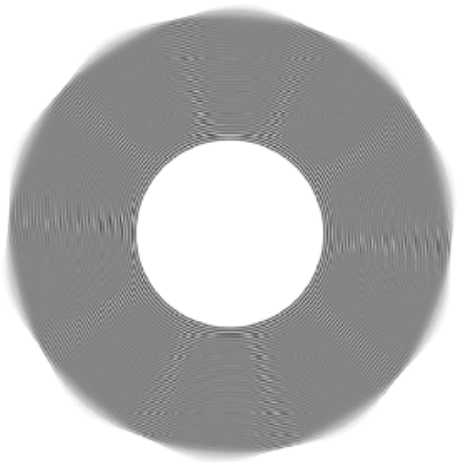

(a)

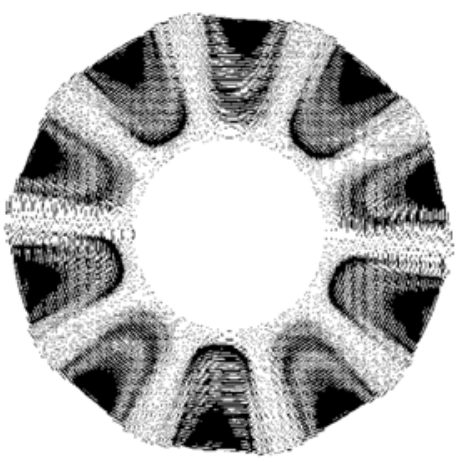

(b)

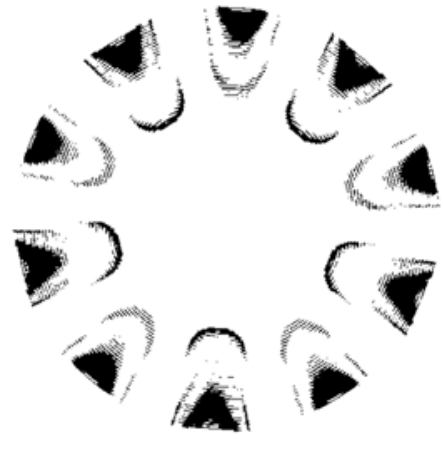

(c)

Figure 10. Visualization of time averaged reflection moiré (chaotic oscillations):

(a) original image; (b) highlighted image; (c) highlighted and cleaned mage

The same sharpening techniques are also applied for images produced by time averaged geometric moiré (Figure 7). Chaotic oscillations (Figure 8) and sufficiently long exposure time $T$ was used for the simulation of original digital image. The value of $k$ is smaller $(k=0.03)$ for this case.
Figure 9 illustrates highlighting of the fringes for time averaged reflection moiré pattern constructed for centrally clamped circular disk oscillating harmonically according to the tenth eigenmode [7]. Analogously, the highlighted images of centrally clamped circular disk oscillating chaotically are given in Figure 10. 
Visualization procedures for time averaged reflection moiré patterns are the same as in the previous examples. Parameter $k$ is equal to 0.07 and 0.03 for harmonic and chaotic oscillations, respectively.

\section{Concluding remarks}

The paper presents some techniques for sharpening pattern of fringes and removing irrelevant moiré grating in digitized moiré images. The proposed visualization procedures help to highlight fringes and interpret the digital moiré images with greater precision, what is essential in hybrid numerical experimental environments.

\section{REFERENCES}

[1] V.A. Deason, J.S. Epstein, A. Abdallah. Dynamic Diffraction Moiré: Theory and Applications. Optics and Lasers in Engineering, 1990, 12 (2-3), 173-87.

[2] R. Gonzalez, R. Woods. Digital Image Processing, Addison-Wesley Publishing Company, 1992.

[3] R. Haralick, L. Shapiro. Computer and Robot Vision, Vol.1, Addison-Wesley Publishing Company, 1992.

[4] R. Klette, P. Zamperoni. Handbook of Image Processing Operators. John Wiley \& Sons Ltd., 1996, 397.

[5] A.S. Kobayashi. Handbook on Experimental Mechanics. 2nd ed. SEM, Bethel, 1993.
[6] M.T. Kokaly, J. Lee, A.S. Kobayashi. Moiré Interferometry for Dynamic Fracture Study. Optics and Lasers in Engineering, 2003, 40 (4), 231-47.

[7] V. Kravcenkiene, L. Saunoriene, M. Ragulskis, A. Palevicius, L. Ragulskis. Directional Conjugate Smoothing of Reflection Moire Images. Information Technology and Control, 2005, 34(2), 120-124.

[8] V. Ostasevicius, M. Ragulskis, A. Palevicius, V. Kravcenkiene, G. Janusas. Applicability of Holographic Technique for Analysis of Non-Linear Dynamics of MEMS Switch. Proceedings of SPIE : Smart Structures and Materials 2005: Smart Electronics, $M E M S$, BioMEMS, and Nanotechnology, Bellingham, 2005, 5763, 405-413.

[9] D. Post, B. Han, P. Ifju. High Sensitivity Moiré: Experimental Analysis for Mechanics and Materials. Springer Verlag, Berlin, 1997.

[10] M. Ragulskis, L. Ragulskis, R. Maskeliunas. Applicability of Time Average Geometric Moiré for Vibrating Elastic Structures. Experimental Techniques, 2004, 28(4), 27-30.

[11] M. Ragulskis, R. Maskeliunas, L. Ragulskis, V. Turla. Investigation of Dynamic Displacements of Lithographic Press Rubber Roller by Time Average Geometric Moiré. Optics and Lasers in Engineering, 2005, 43, 951-962.

Received May 2006. 